\title{
Simulation of Daily Precipitation from CMIP5 in the Qinghai-Tibet Plateau
}

\author{
Feng Zhang ${ }^{1,4}$, Hang Ren ${ }^{1}$, Lijuan Miao ${ }^{2,3}$, Yadong Lei ${ }^{1}$, and Mingkeng Duan ${ }^{1}$ \\ ${ }^{1}$ Key Laboratory of Meteorological Disaster, Ministry of Education (KLME)/ \\ Collaborative Innovation Center on Forecast and Evaluation of Meteorological Disaster (CIC-FEMD), \\ Nanjing University of Information Science and Technology, Nanjing, China \\ ${ }^{2}$ Collaborative Innovation Center on Forecast and Evaluation of Meteorological Disasters, \\ School of Geographical Sciences, Nanjing University of Information Science and Technology, Nanjing, China \\ ${ }^{3}$ Department of Structural Development of Farms and Rural Areas, \\ Leibniz Institute of Agricultural Development in Transition Economies, Halle (saale), Germany \\ ${ }^{4}$ State Key Laboratory of Severe Weather, Chinese Academy of Meteorological Sciences, Beijing, China
}

\begin{abstract}
As the earth's third pole, Qinghai-Tibet Plateau belongs to one of the most sensitive regions to climate change in the world. Based on the observed and the simulated daily precipitation from the Coupled Model Intercomparison Project Phase 5 (CMIP5), we evaluated the simulation performance of daily precipitation from selected CMIP5 models from 1975 to 2005 over the Qinghai-Tibet Plateau. We found that daily precipitation exhibited obvious longrange correlation characteristics using the detrended fluctuation analysis method. The scaling exponents of daily precipitation in summer and autumn are significantly larger than those in spring and winter. MIROC4H with the best performance can reproduce long-range correlation characteristic of daily precipitation series probably because of the higher resolution, which can capture small scale cloud convections. Besides there are seasonal differences in the simulation results among different regions of the QinghaiTibet Plateau, simulation effects of all climate models in summer and winter are better than those in spring and autumn. The performance of MIROC4H model works the best in spring. Overall, the scaling exponents of daily precipitation from BCC-CSM1-1-M, CMCC-CM and MIROC4H are close to the observations. CCSM4 and MIROC4H climate models could reproduce the internal dynamics characteristic of daily precipitation in autumn. But for winter, all climate models have exaggerated the scaling value in southeastern Qinghai-Tibet Plateau compared with the observed values.
\end{abstract}

(Citation: Zhang, F., H. Ren, L. Miao, Y. Lei, and M. Duan, 2019: Simulation of daily precipitation from CMIP5 in the QinghaiTibet Plateau. SOLA, 15, 67-73, doi:10.2151/sola.2019-014.)

\section{Introduction}

Climate system models are important tools for simulating and estimating climate change. The simulation results have been widely applied in hydrological, ecological, environmental and agricultural fields (Parry et al. 2004; Fowler et al. 2007; Jacob and Winner 2009). However, simulation results of climate models still have uncertainty due to the complexity of the climate system. Therefore, how to evaluate simulation performance of climate models objectively and quantitatively is of great scientific significance and practical value for future climate predictions (Masson and Knutti 2011). A large amount of research work has been done on the quantitative evaluation of performance of CMIP5 models, which has received considerable attention in recent year (Perkins et al. 2007; Chen et al. 2011; Song and Zhou 2014).

Traditional assessment methods of climate models are mainly based on the differences of the mean, variance, trend and other

Corresponding author: Lijuan Miao, Nanjing University of Information Science and Technology, 210044, No. 19 Ningliu Road, Nanjing, China. E-mail: miaolijuan1111@gmail.com. aspects of simulation variables between simulated values and observed values. However, the climate system is a highly complex, dissipative, and non-adiabatic nonlinear dynamical system, and the time series of meteorological elements are also nonstationary. The mean, variance, and trend of these series are all changing over time. Therefore, traditional assessment methods of climate models have some shortcomings. It is noteworthy that, in recent years, results of nonlinear research have shown that changes in the climate system have self-memory. That is to say, past climate evolution has a long-term effect on the future evolution of the climate system (Eichner et al. 2003; Monetti et al. 2003). This effect is the so-called "long-term memory" or "scale" in nonlinear science. Detrended fluctuation analysis (DFA), a non-linear method of scale analysis, can effectively filter out the strong trend components caused by external forcing in the time series, and excavate the fluctuation components induced by the long-range correlation. Due to its advantages and applicability (Bashan et al. 2008), this method has been widely used in the meteorological field in recent years (Kantelhardt et al. 2006; Fraedrich et al. 2009; Zhu et al. 2010; Bunde et al. 2013; Jiang et al. 2015; Yuan et al. 2015; He et al. 2016; Jiang et al. 2017). With the extensive usage of climate models, people begin to use the DFA method to assess merits of climate models by comparing the difference of scaling exponents between the simulated data of climate models and observed data (Govindan et al. 2002; Zhao and He 2015).

The Qinghai-Tibet Plateau, known as the third pole, has an average elevation of over 4,000 meters. Its precipitation changes have a significant impact on the economy and water resources of local and neighboring countries (Cui et al. 2007; Xu et al. 2008; Immerzeel et al. 2010). Previous studies have shown that changes in precipitation in the eastern part of the Qinghai-Tibet Plateau is mainly due to the oceanic water vapor caused by the Asian monsoon circulation, while the Asian monsoon circulation is regulated by El Niño Southern Oscillation (ENSO), Indian Ocean Dipole Mode (IOD) and North Altantic Oscillation (NAO) (Ashok, Guan and Yamagata 2001; Cherchi and Navarra 2013; Fasullo and Webster 2002; Liu et al. 2015; Liu et al. 2016; Liu and Yin 2001). In addition, climate change in the Qinghai-Tibet Plateau has been investigated based on the observational data analysis in earlier times. However, the observational data in the region is relatively scarce due to the limitations of natural conditions. In recent years, much more climate models have been applied to the study of climate change in the Qinghai-Tibet Plateau with the development of computer technology. Therefore, how to accurately simulate the precipitation characteristics of the Qinghai-Tibet Plateau is a persistent concern of domestic and foreign scholars.

In this study, the DFA method is used to analyze the longrange correlation behaviors of daily precipitation series over the Qinghai-Tibet Plateau, and the precipitation simulation capability of 9 global climate models over the Qinghai-Tibet Plateau is evaluated based on the intrinsic dynamics of precipitation. 


\section{Data and study method}

\subsection{Data}

The precipitation data from the China Meteorological Administration are used to evaluate precipitation simulation derived from climate models. It was from the interpolation of the daily observational records of 2416 Chinese meteorological stations with $0.5 \times$ 0.5 horizontal resolution. The data cover daily data from 1975 to 2005. Table 1 shows details of 9 CMIP5 models adopted for longterm experiment in this study. A detailed description of this experiment was given by Taylor et al. (2012) (Taylor et al. 2012). Daily precipitation data obtained from the historical experiment from 1975 to 2005 are used in this study. For convenient quantitative evaluation of the difference in long-range correlation characteristic between observed data and simulated data, scaling exponents from climate models are interpolated to the grid data with $0.5 \times 0.5$ horizontal resolution using a bilinear interpolation method.

\subsection{The DFA methodology}

Detrend fluctuation analysis (DFA) method (Peng et al. 1994) is the revision and perfection of the traditional fluctuation analysis technique and the rescaled range analysis (Hurst 1951) based on the random walk theory. A brief description of some of the steps of DFA is given as follows:

(1) The cumulative deviation series $\{y(j), j=1,2, \ldots, N\}$ of the original time series (with annual cycle removed) $\{x(i), i=$ $1,2, \ldots, N\}$ is calculated:

$$
y(j)=\sum_{i=1}^{i=j} \Delta x(i) \quad(j=1,2, \ldots, N)
$$

where and $\Delta x(i)=x(i)-\langle x\rangle$ and $\langle x\rangle$ is the mean of raw time series $\{x(i), i=1,2, \ldots, N\}$.

(2) The cumulative deviation series is divided into $\mathrm{m}$ windows by equal length $s$, where $s$ is the interval length, i.e. the time scale; $m$ is the number of windows with an integer part of $N / s$. In order to make full use of the data left over from the tail, the cumulative deviation series is re-divided from the back. Then we will get $2 m$ windows $v=1,2, \ldots, 2 m$ and the time series of each window is recorded as $z(v, j)$.

(3) The local trend $p(v, j)$ in each segment is obtained with the least squares method. In this paper, we use 2-order polynomial, i.e. DFA2 (Bunde et al. 2000; Kantelhardt et al. 2001). Then the local trend is subtracted in each window.

$$
\begin{aligned}
& Q(v, j)=z(v, j)-p(v, j) \\
& \quad(v=1,2, \ldots, m ; j=1,2, \ldots, s) \\
& R(v, j)=z(v, j)-p(v, j) \\
& \quad(v=m+1, m+2, \ldots, 2 m ; j=1,2, \ldots, s)
\end{aligned}
$$

Among them, $Q(v, j)$ and $R(v, j)$ represent dividing the data into windows from front to back and from back to front, respectively.
(4) The fluctuation function in each segment is calculated.

$$
H(v, s)= \begin{cases}\frac{1}{s} \sum_{j=1}^{s} Q^{2}(v, j) & (v=1,2, \ldots, m) \\ \frac{1}{s} \sum_{j=1}^{s} R^{2}(v, j) & (v=m+1, m+2, \ldots, 2 m)\end{cases}
$$

(5) The fluctuation function $F(s)$ is obtained with the arithmetic mean of the fluctuation function in all segments.

$$
F(s)=\left[\frac{1}{2 m} \sum_{v=1}^{2 m} H(v, s)\right]^{1 / 2}
$$

The fluctuation function $F(s)$ changes with the window size $s$. In general, $F(s)$ increases when the window size $s$ increases. The fluctuation function $F(s)$ and the window size $s$ satisfy the powerlaw relationship $F(s) \sim s^{\alpha}$ if the time series exhibits long-range correlation characteristic. The slope of the double logarithmic curve for $F(s)$ and $s$ represents the scaling exponent of time series. If $0.5<\alpha<1(0<\alpha<0.5)$, the time series exhibits a positive (negative) long-range correlation characteristic. If $\alpha=0.5$, the time series is uncorrelated. In addition, the long-range correlation characteristic of a given climate variability can be quantitatively described by using the DFA method. However, there will be some uncertainties due to the length of the observed data. In 2015, Zhao and He produced 20000 artificial series with the given scaling exponents by Fourier Filtering in order to test the uncertainties of DFA method. Results of the artificial series indicate that the uncertainties of DFA method range from -0.05 to 0.05 for the precipitation series (Zhao and He 2015). For convenience, this article defines the deviation ranging from -0.05 to 0.05 as the 0.1 significant test level of precipitation series over the Qinghai-Tibet Plateau.

\section{Results}

\subsection{Evaluation of simulations for precipitation series}

Surface precipitation is one of the most important and essential meteorological elements as well as the climate change indicator of most concern (Huang et al. 2018). Previous studies have shown that the evolution of global precipitation exhibits a long-range correlation characteristic (Zhao and He 2015; Jiang et al. 2017). Considering the complex effects of the Asian monsoon system and the terrain of the Qinghai-Tibet Plateau(between $25^{\circ} \mathrm{N}-40^{\circ} \mathrm{N}$ and $75^{\circ} \mathrm{E}-105^{\circ} \mathrm{E}$ ), does precipitation over the Qinghai-Tibet Plateau also have long-range correlation characteristic? And if it has, can the existing global climate models reproduce the long-range correlation characteristic? In this paper, the DFA technique is applied to evaluate simulated precipitation on the Qinghai-Tibet Plateau from 1975 to 2005 . Figure 1 shows the time series of the average precipitation over the Qinghai-Tibet Plateau by double logarithmic

Table 1. Information of 9 CMIP5 climate models (The unit of spatial resolution: longitudinal grid points $\times$ latitudinal grid points).

\begin{tabular}{llr}
\hline \multicolumn{1}{c}{ Model Name } & \multicolumn{1}{c}{ Institute (Country) } & $\begin{array}{c}\text { Grid points } \\
\text { (Longitude } \times \text { Latitude) }\end{array}$ \\
\hline ACCESS1-3 & Commonwealth Scientific and Industrial Research Organisation (Australia) & $192 \times 145$ \\
BCC-CSM1-1-m & Beijing Climate Center, China Meteorological Administration (China) & $320 \times 160$ \\
CCSM4 & National Center for Atmospheric Research (USA) & $288 \times 192$ \\
CESM1-CAM5 & National Science Foundation, Department of Energy, National Center for Atmospheric Research & $288 \times 192$ \\
CMCC-CM & Centro Euro-Mediterraneo per I CambiamentiClimatici (Italy) & $480 \times 240$ \\
CNRM-CM5 & Centre National de RecherchesMeteorologiques/Centre Europeen de Recherche et Formation & $256 \times 128$ \\
& Avancees en CalculScientifique (France) & $320 \times 160$ \\
EC-EARTH & EC-EARTH consortium & $640 \times 320$ \\
MIROC4h & Atmosphere and Ocean Research Institute (The University of Tokyo), National Institute for Environmental & \\
& Studies, and Japan Agency for Marine-Earth Science and Technology (Japan) & $320 \times 160$ \\
MRI-CGCM3 & Meteorological Research Institute (Japan) & \\
\hline
\end{tabular}



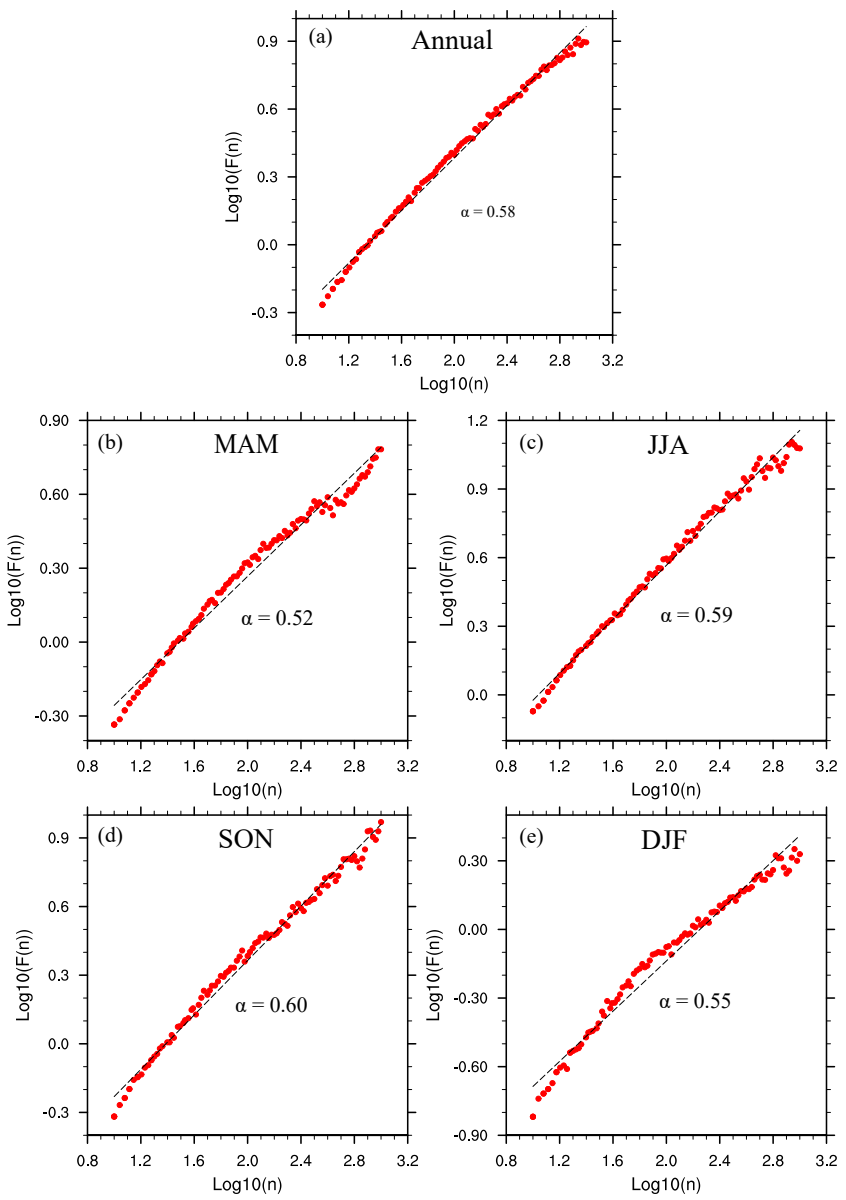

Fig. 1. The DFA analysis result for annual (a) and four seasons $(b-e)$ regional mean precipitation in the Qinghai-Tibet Plateau from 1975 to 2005 , the red solid point represents the obtained fluctuation function; the black dashed line represents the linear fitting line of the red solid point, and the slope can be a good index for long-range correlation characteristic on this time scale).

curve of the fluctuation function. Through linear fitting, we found that the annual precipitation in the Qinghai-Tibet Plateau exhibit relatively weak long-range correlation characteristic with scaling exponents of 0.58 . And the scaling exponents for precipitation in four seasons are $0.52,0.59,0.60$ and 0.55 , respectively. More obvious long-range correlation characteristic is showed in summer and autumn, but the scaling exponents are close to 0.5 in spring and winter, thus showing uncorrelation.

Then we evaluated the simulation performance of precipitation by calculating the difference of scaling exponents between simulated data and observed data. Figure 2 shows the results of the DFA based on the 9 CMIP5 models. Interestingly, the simulated scaling exponents of all climate models are larger than those of observations. The scaling exponents simulated by ACCESS1-3, CCSM4, CESM1-CAM5 and MIROC4H are 0.61, 0.60, 0.61 and 0.59 , respectively, which pass the $90 \%$ significance test. It means that the four global climate models (e.g., ACCESS1-3, CCSM4, CESM1-CAM5 and MIROC4H) can reproduce the intrinsic variation characteristics of regional average precipitation in the Qinghai-Tibet Plateau. In addition, simulation results of all models are close to observations, and there are 7,6 and 4 climate models passing the $90 \%$ significance test in summer, autumn and winter, respectively. It is further revealed that the long-range correlation characteristic of regional mean precipitation over the QinghaiTibet Plateau can be better simulated in summer, autumn and winter by all climate models. However, in spring the simulated scaling exponent is significantly larger and there are no models
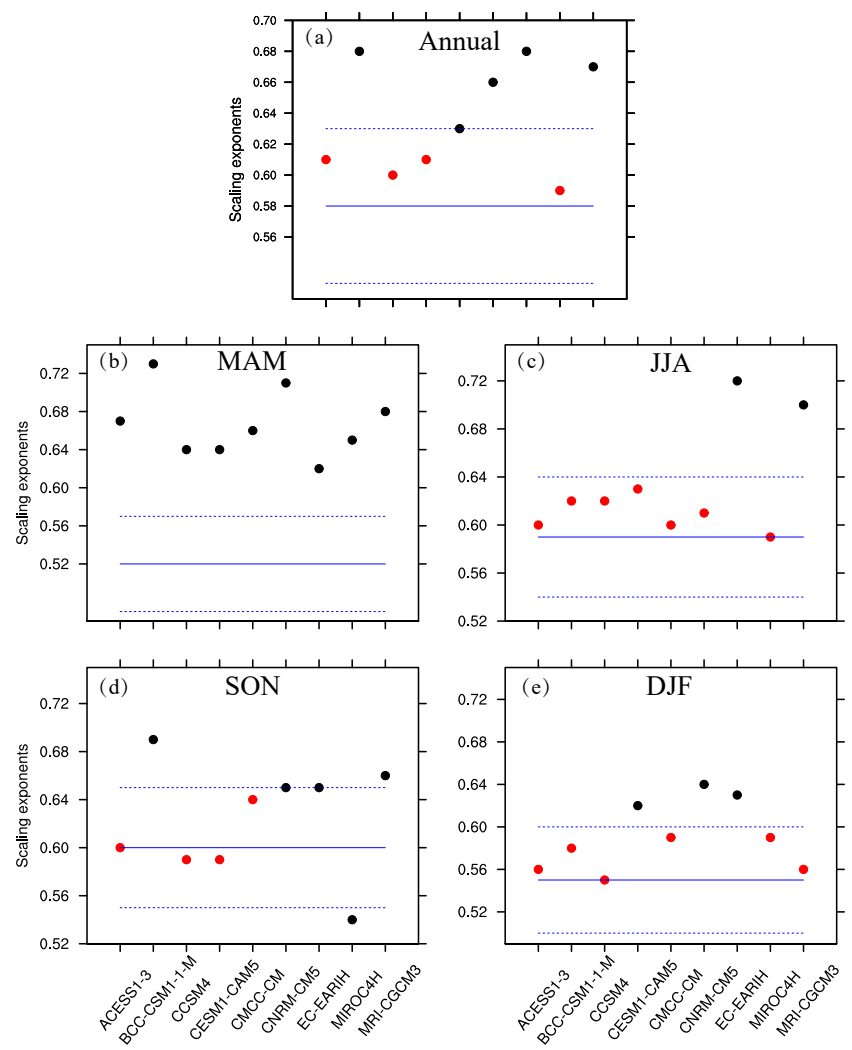

Fig. 2. The DFA2 analysis results of 9 climate models for the annual (a) and four seasons $(\mathrm{b}-\mathrm{e})$ regional mean precipitation in the Qinghai-Tibet Plateau. The blue solid line represents the scaling exponent in observation data while the blue dashed line is the $90 \%$ confidence level. The red point indicates that the simulation result passes the $90 \%$ significance test.

passing the significance test. In addition, simulation result of the EC-EARTH climate model is the worst among all climate models since it doesn't pass the $90 \%$ significance test for all four seasons.

\subsection{Spatial evaluation in precipitation simulations}

Figure 3 shows the spatial distribution of scaling exponents of the precipitation series for observation (a) and simulation $(b-j)$ over the Qinghai-Tibet Plateau. Figure 3a indicates that scaling exponents of precipitation show a decreasing trend from the southern part to the northern part of Qinghai-Tibet Plateau. Scaling exponents of precipitation on the northeastern Qinghai-Tibet Plateau are between 0.48 and 0.52 , indicating that the precipitation is uncorrelated; in the southern Qinghai-Tibet Plateau scaling exponents of precipitation are more than 0.55 , thus exhibiting a weak long-range correlation characteristic. This distribution feature is closely related to the climatic conditions of the QinghaiTibet Plateau. Precipitation over the south Qinghai-Tibet Plateau is mainly due to the oceanic water vapor caused by the southwest monsoon, while precipitation in the north part is mainly attributed to the re-evaporation of surface water, which is produced by the inland circulation system. Compared with the observations, simulated scaling exponents of all climate models are close to those of the observation in the central and eastern Qinghai-Tibet Plateau, and are significantly larger in the western part. CNRM-CM5, ECEARTH and MRI-CGCM3 climate models have the worst simulation results (less than $40 \%$ of the grid pass the $90 \%$ significance test), with simulation errors being greater than 0.1 for the middle and eastern Qinghai-Tibet Plateau.

To quantitatively evaluate the seasonal variation of spatial distribution of scaling exponents over the Qinghai-Tibet Plateau, the DFA method is applied to simulation the precipitation in four seasons. The analysis results are shown from Fig. 4 to Fig. 5, and details of errors between observation and simulation are shown 
(a) Observation

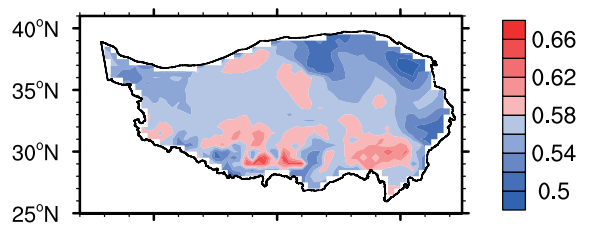

(c) BCC-CSM1-1-m

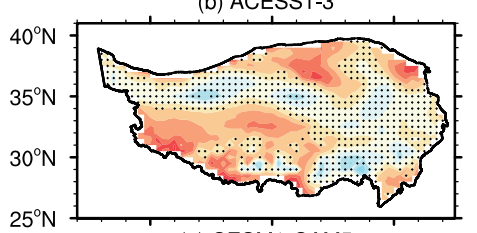

(e) CESM1-CAM5

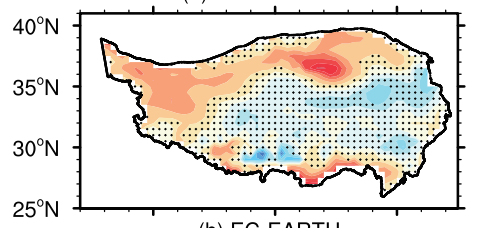

(h) EC-EARTH
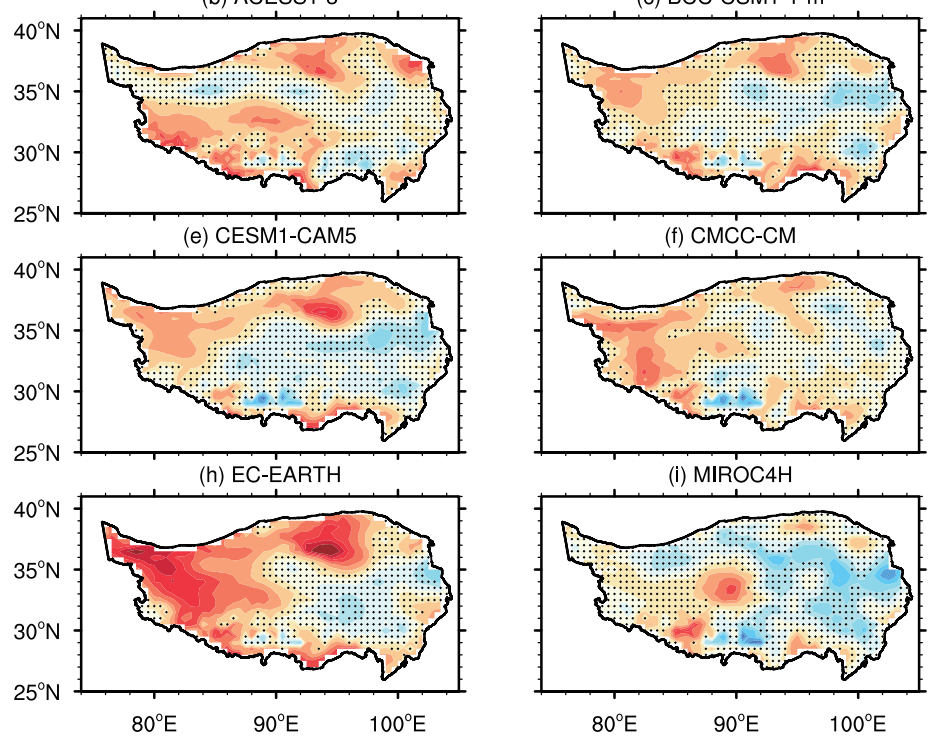

(f) CMCC-CM

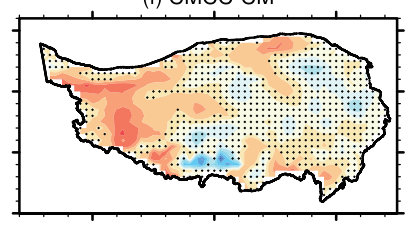

(i) $\mathrm{MIROC} 4 \mathrm{H}$
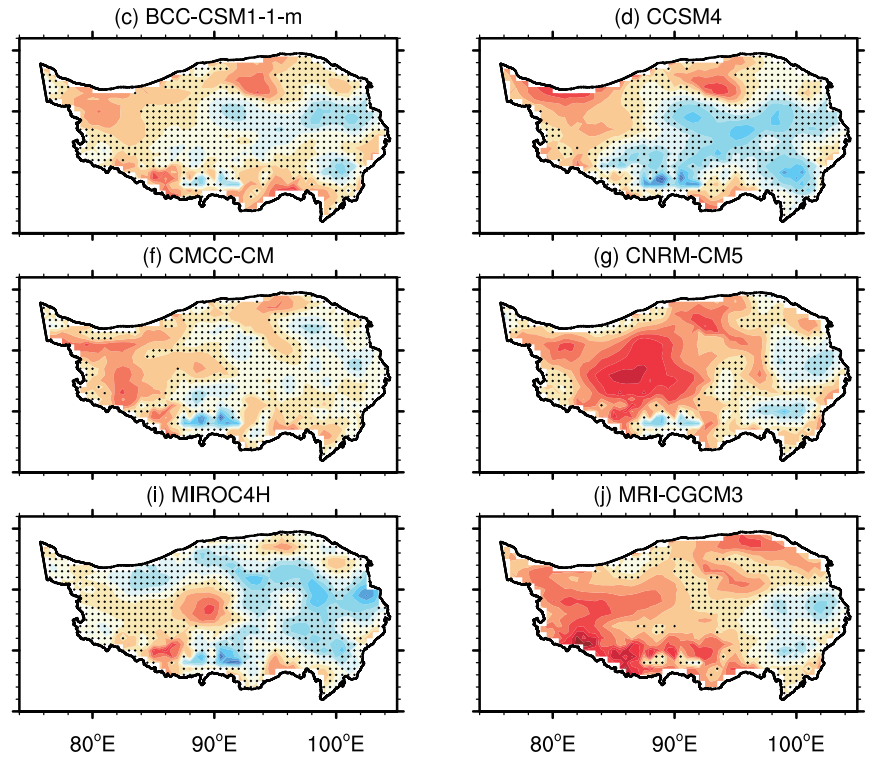

(g) CNRM-CM5

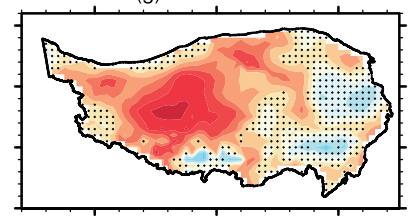

(j) MRI-CGCM3

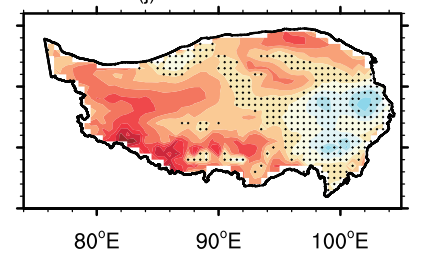

$0.12 \quad 0.16$

Fig. 3. The spatial distribution of scaling exponents for observation and simulation of annual regional mean precipitation in the Qinghai-Tibet Plateau. (a) Observation; $(b-j)$ simulation minus observation. The black dot indicates that the simulation passes the $90 \%$ significance test.

(a) MAM

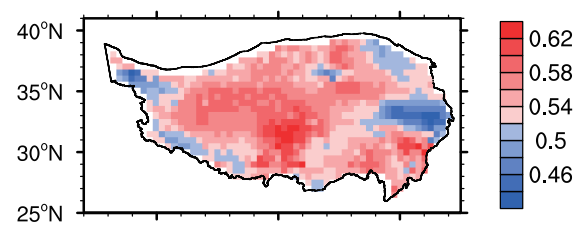

(c) $\mathrm{SON}$

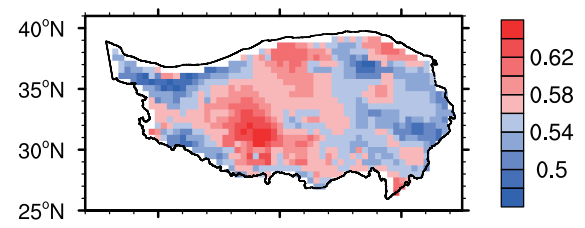

(b) JJA

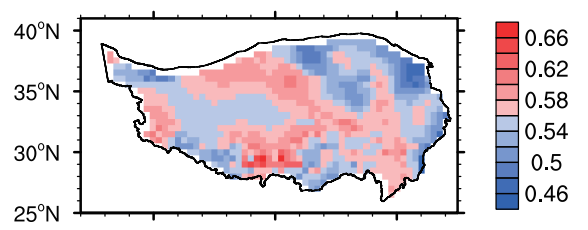

(d) DJF

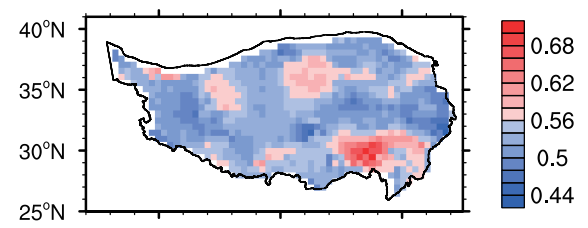

Fig. 4. The spatial distribution of scaling exponents for observation in four seasons.(a) Spring; (b) Summer; (c) Autumn; (d) Winter.

in Table 2. In spring (Fig. 4a), scaling exponents of precipitation series over the Qinghai-Tibet Plateau are evenly distributed and mainly concentrated in $0.54-0.62$, showing obvious long-range correlation characteristic. Compared with the observation, scaling exponents of ACCESS1-3, CMCC-CM and CNRM-CM5 climate models are significantly larger in the western part of the QinghaiTibet Plateau, and only $35 \%, 40 \%$ and $26 \%$ of the grid pass the $90 \%$ significance test, respectively. The MIROC4H climate model has the best simulation effect with $63 \%$ of the grid passing the significance test, indicating that it can reproduce the long-range correlation characteristic of precipitation series over the central Qinghai-Tibet Plateau (Fig. 5(1-9)). This is probably because of the higher resolution of the model, which can capture small scale cloud convections. In summer (Fig. 4b), scaling exponents of precipitation are close to 0.5 in the northeast and southwest of the Qinghai-Tibet Plateau, showing uncorrelation. Scaling exponents are between 0.58 and 0.62 on the central Qinghai-Tibet Plateau, demonstrating long-range correlation characteristic and indicating strong correlation. The simulated scaling exponents of BCCCSM1-1-M, CMCC-CM and MIROC4H climate models are close to those of observations, and $65 \%, 62 \%$ and $60 \%$ of the grid pass the significance test, respectively. The simulated scaling exponents of CESM1-CAM5, CNRM-CM5, EC-EARTH, and MRI-CGCM3 climate models are significantly larger in the western part of the 
Table 2. The simulation errors between each climate model and observation in spatial distribution in four seasons.

\begin{tabular}{|c|c|c|c|c|c|c|}
\hline \multirow{2}{*}{ Model } & \multicolumn{3}{|c|}{ Spring } & \multicolumn{3}{|c|}{ Summer } \\
\hline & $\Delta \alpha>0.05$ & $-0.05<\Delta \alpha<0.05$ & $\Delta \alpha<-0.05$ & $\Delta \alpha>0.05$ & $-0.05<\Delta \alpha<0.05$ & $\Delta \alpha<-0.05$ \\
\hline ACCESS1-3 & $61 \%$ & $35 \%$ & $4 \%$ & $38 \%$ & $58 \%$ & $4 \%$ \\
\hline BCC-CSM1-1-m & $52 \%$ & $45 \%$ & $3 \%$ & $22 \%$ & $65 \%$ & $13 \%$ \\
\hline CCSM4 & $51 \%$ & $44 \%$ & $5 \%$ & $28 \%$ & $47 \%$ & $25 \%$ \\
\hline CESM1-CAM5 & $45 \%$ & $52 \%$ & $3 \%$ & $37 \%$ & $50 \%$ & $13 \%$ \\
\hline CMCC-CM & $59 \%$ & $40 \%$ & $1 \%$ & $30 \%$ & $62 \%$ & $8 \%$ \\
\hline CNRM-CM5 & $73 \%$ & $26 \%$ & $1 \%$ & $52 \%$ & $44 \%$ & $4 \%$ \\
\hline EC-EARTH & $43 \%$ & $48 \%$ & $9 \%$ & $57 \%$ & $37 \%$ & $6 \%$ \\
\hline MIROC4h & $26 \%$ & $63 \%$ & $11 \%$ & $21 \%$ & $60 \%$ & $19 \%$ \\
\hline \multirow[t]{3}{*}{ MRI-CGCM3 } & $52 \%$ & $44 \%$ & $4 \%$ & $67 \%$ & $32 \%$ & $1 \%$ \\
\hline & \multicolumn{3}{|c|}{ Autumn } & \multicolumn{3}{|c|}{ Winter } \\
\hline & $\Delta \alpha>0.05$ & $-0.05<\Delta \alpha<0.05$ & $\Delta \alpha<-0.05$ & $\Delta \alpha>0.05$ & $-0.05<\Delta \alpha<0.05$ & $\Delta \alpha<-0.05$ \\
\hline ACCESS1-3 & $34 \%$ & $57 \%$ & $9 \%$ & $34 \%$ & $58 \%$ & $8 \%$ \\
\hline BCC-CSM1-1-m & $55 \%$ & $44 \%$ & $1 \%$ & $38 \%$ & $51 \%$ & $11 \%$ \\
\hline CCSM4 & $26 \%$ & $67 \%$ & $7 \%$ & $26 \%$ & $55 \%$ & $19 \%$ \\
\hline CESM1-CAM5 & $37 \%$ & $53 \%$ & $10 \%$ & $45 \%$ & $45 \%$ & $10 \%$ \\
\hline CMCC-CM & $40 \%$ & $52 \%$ & $8 \%$ & $37 \%$ & $51 \%$ & $12 \%$ \\
\hline CNRM-CM5 & $62 \%$ & $33 \%$ & $5 \%$ & $69 \%$ & $28 \%$ & $3 \%$ \\
\hline EC-EARTH & $32 \%$ & $55 \%$ & $13 \%$ & $73 \%$ & $23 \%$ & $4 \%$ \\
\hline MIROC4h & $16 \%$ & $67 \%$ & $17 \%$ & $38 \%$ & $51 \%$ & $11 \%$ \\
\hline MRI-CGCM3 & $30 \%$ & $59 \%$ & $11 \%$ & $28 \%$ & $59 \%$ & $13 \%$ \\
\hline
\end{tabular}

Qinghai-Tibet Plateau (Fig. 5(10-18)). In autumn (Fig. 4c), scaling exponents of the precipitation series in the eastern part of the Qinghai-Tibet Plateau are between 0.48 and 0.52, showing uncorrelation, while those in the western region are between 0.56 and 0.62 , exhibiting long-range correlation characteristic and strong correlation. Compared with the observation, the CCSM4 and MIROC4H climate models can simulate the internal dynamics characteristic of precipitation over the Qinghai-Tibet Plateau, with $67 \%$ of the grid of both models passing the $90 \%$ significance test. Scaling exponents of the ACCESS1-3 climate model are larger in the southwest of the Qinghai-Tibet Plateau (Fig. 5(19-27)). In winter (Fig. 4d), precipitation series in most parts of the QinghaiTibet Plateau show strong uncorrelation, and the scaling exponents are close to 0.5 ; scaling exponents of the southeastern region are between 0.62 and 0.68 , showing correlation. The simulation results indicate that simulated scaling exponents of all climate models are larger than those of observations over the southeast Qinghai-Tibet Plateau. In addition, simulation results of the CNRM-CM5 and EC-EARTH climate models are the worst (only $28 \%$ and $23 \%$ of the grid points pass the significance test respectively), and the simulation errors are above 0.14 in the middle of the Qinghai-Tibet Plateau (Fig. 5(28-36)).

\section{Discussion}

Previous studies demonstrate that climate models have poor performance in simulating precipitation over the Qinghai-Tibet Plateau. Although most climate models are able to reasonably reproduce the spatial pattern of annual precipitation increasing from northwest to southeast, all of them tend to overestimate the precipitation in climatological annual and seasonal means $(\mathrm{Su}$ et al. 2013; Hu et al. 2014). In this study, we evaluate the performance of 9 climate models used in CMIP5 in simulating the longrange correlation characteristics for precipitation over the QinghaiTibet Plateau. Simulation results reveal that climate models have better performance in simulating the long-range correlation characteristics for precipitation in the middle and eastern part of the Qinghai-Tibet Plateau than the western region. For errors between simulation and observation in the eastern part of the Qinghai-Tibet Plateau, on the one hand, long-term climate change characteristics can be simulated on the global and hemispheric scale but the dramatic changes of climatic factors cannot be captured due to low spatial resolution $(110 \mathrm{~km})$ of the climate models. There are few descriptions of complex local terrain conditions and microphysical processes. On the other hand, it is possible that the climate models focus on the long-term changes caused by external forcing (greenhouse gases, aerosols, solar constants, etc.) of the historical climate, and don't pay enough attention to the interannual and interdecadal natural variability within the climate system (ENSO, IOD, and NAO). For the difference between simulation and observation over the western Qinghai-Tibet Plateau, one explanation is that precipitation in the climate model is calculated by the microphysics and cumulus convective parameterization schemes. So we suggest the errors between observation and simulation probably induced by the improper microphysics and cumulus convective parameterization schemes due to the complex terrain over the Qinghai-Tibet Plateau. Besides, since meteorological stations are sparse due to the complex terrain of the western Qinghai-Tibet Plateau, there is a certain amount of errors in the observed data. Therefore, the difference of scale behaviors between observed data and simulated data mainly reflects the internal problems of the models, such as defects in the microphysics parameterization, insufficient resolution of the models or lack of perfect terrain processing. Analysis of these error characteristics can help to better understand the development and limitations of the models and further improve the climate models' simulation capability for precipitation over the Qinghai-Tibet Plateau.

\section{Conclusion}

As the earth's third pole, Qinghai-Tibet Plateau belongs to one of the most sensitive regions to climate change in the world. In this paper, the DFA method is used to analyze the long-term correlation of daily precipitation series over the Qinghai-Tibet Plateau based on observed and the simulated daily precipitation from the CMIP5. The major conclusions are summarized as follows.

(1) The precipitation time series (regional average) over the Qinghai-Tibet Plateau exhibit relatively weak long-range correlation characteristic with scaling exponents of 0.58 . And the scaling exponents show a decreasing trend from the south to the north Qinghai-Tibet Plateau.

(2) Simulation results reveal that the scaling exponents of precipitation of all climate models are close to those of observations in the middle and eastern part of the Qinghai-Tibet Plateau 
MAM
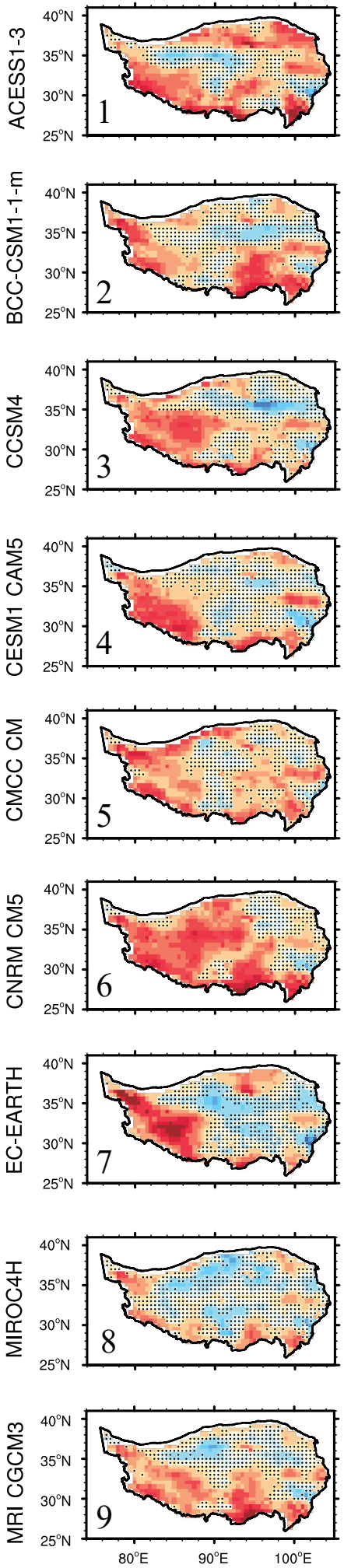

JJA
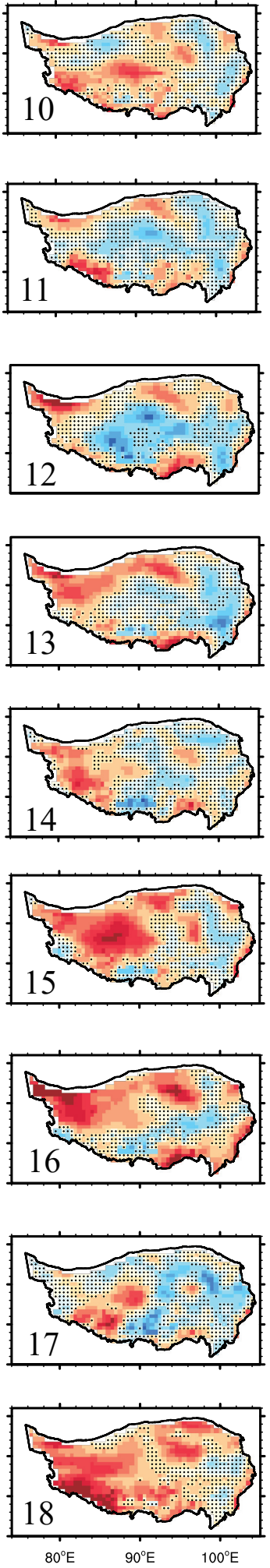

SON
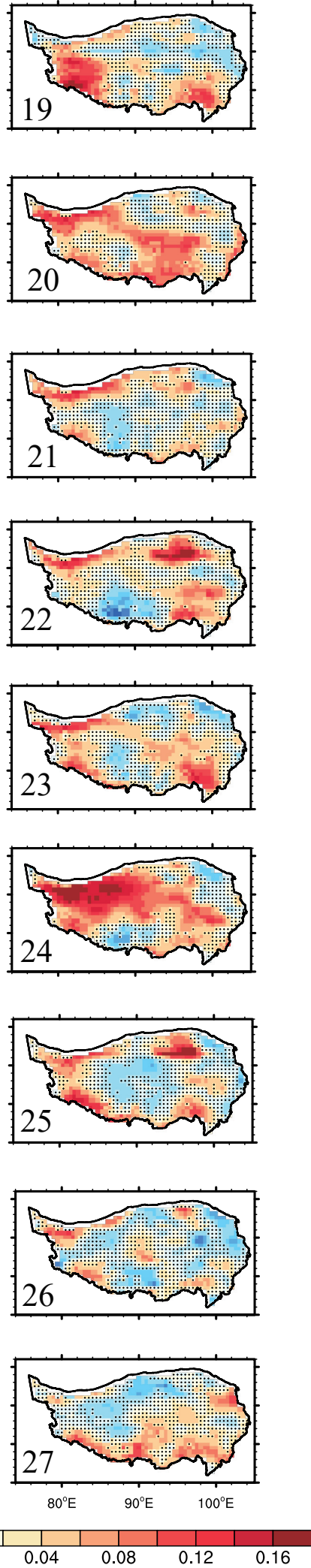

DJF
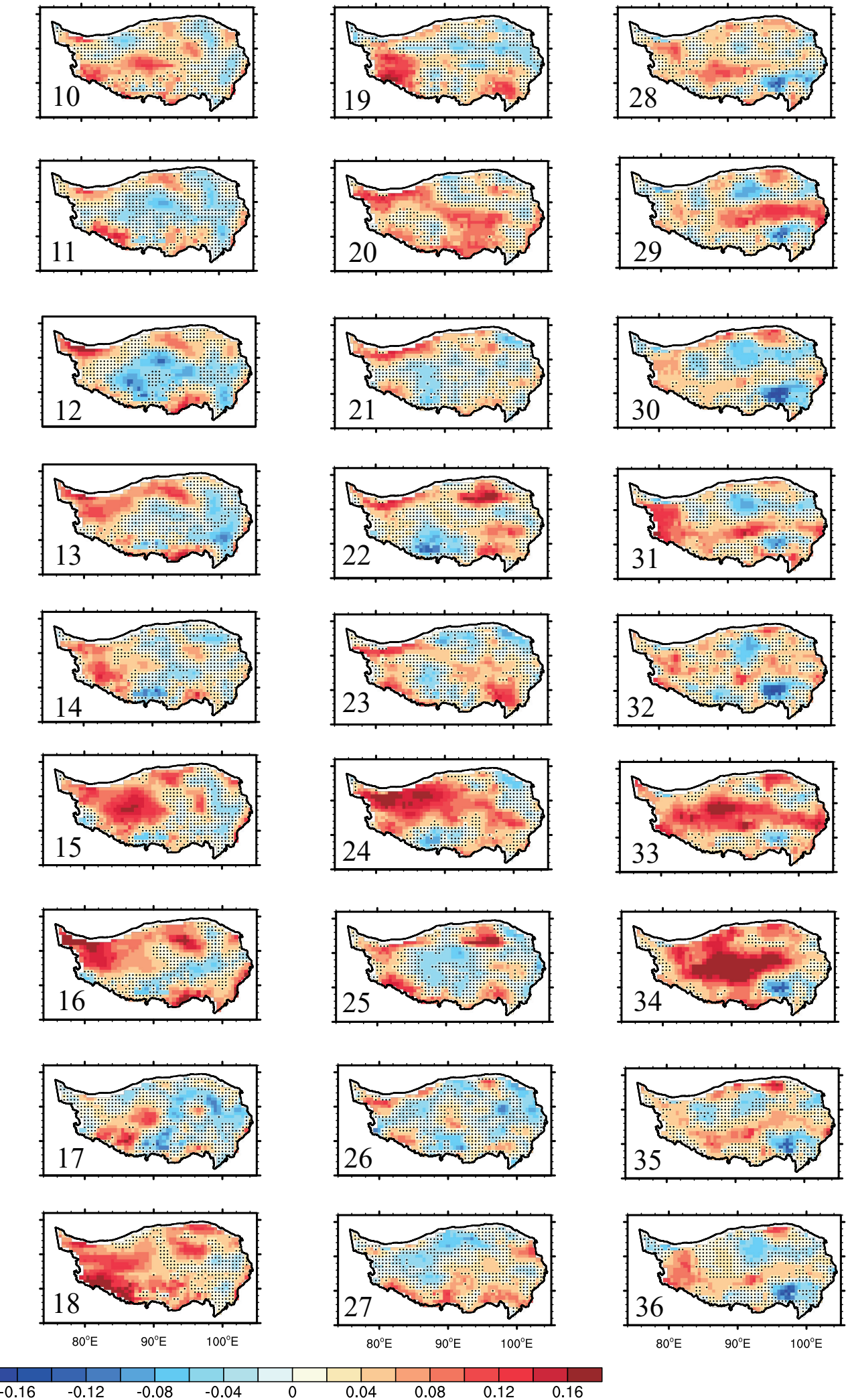

Fig. 5. The spatial distribution of scaling exponents for simulation of precipitation in the Qinghai-Tibet Plateau in four seasons. (1-9) Spring; (10-18) Summer; (19-27) Autumn; (28-36) Winter. The black dot indicates that the simulation passes the $90 \%$ significance test. 
and significantly larger in the western region.

(3) There are seasonal differences in the simulation results among different regions of the Qinghai-Tibet Plateau, simulation effects of all climate models in summer and winter are better than those in spring and autumn. The performance of MIROC4H model works the best in spring. In summary, the scaling exponents of daily precipitation from BCC-CSM11-M, CMCC-CM and MIROC4H are close to the observations. CCSM4 and MIROC4H climate models could reproduce the internal dynamics characteristic of daily precipitation in autumn. But for winter, all climate models have exaggerated the scaling value in southeastern Qinghai-Tibet Plateau compared with the observed values.

\section{Acknowledgements}

The work was supported by the National Natural Science Foundation of China (41675003, 41675056), the Key Project of National Social and Scientific Fund Program (16ZDA047), National Key Research and Development Programof China (2017YFC 0504301), and Startup Foundation for Introducing Talentsof Nanjing University of Information Science and Technology (2243141601048).

Edited by: Yu Kosaka

\section{References}

Bashan, A., R. Bartsch, J. W. Kantelhardt, S. Havlin, and I. Applications, 2008: Comparison of detrending methods for fluctuation analysis. Physica A: Stat. Mech., 387, 5080-5090.

Bunde, A., U. Büntgen, J. Ludescher, J. Luterbacher, and H. Von Storch, 2013: Is there memory in precipitation? Nat. Climate Change, 3, 174.

Bunde, A., S. Havlin, J. W. Kantelhardt, T. Penzel, J.-H. Peter, and K. Voigt, 2000: Correlated and uncorrelated regions in heart-rate fluctuations during sleep. Phys. Rev. Lett., 85, 3736.

Chen, W., Z. Jiang, and L. Li, 2011: Probabilistic projections of climate change over China under the SRES A1B scenario using 28 AOGCMs. J. Climate, 24, 4741-4756.

Cui, X., B. Langmann, and H.-F. Graf, 2007: Summer monsoonal rainfall simulation on the Tibetan Plateau with a regional climate model using a one-way double-nesting system. SOLA, 3, 49-52.

Eichner, J. F., B. E. Koscielny, A. Bunde, S. Havlin, and H. J. Schellnhuber, 2003: Power-law persistence and trends in the atmosphere: A detailed study of long temperature records. Phys. Rev., 68, 046133.

Fowler, H. J., S. Blenkinsop, and C. Tebaldi, 2007: Linking climate change modelling to impacts studies: Recent advances in downscaling techniques for hydrological modelling. Int. J. Climatol., 27, 1547-1578.

Fraedrich, K., R. Blender, and X. Zhu, 2009: Continuum climate variability: Long-term memory, scaling, and 1/f-noise. Int. J. Modern Phys., 23, 5403-5416.

Govindan, R., D. Vyushin, A. Bunde, S. Brenner, S. Havlin, and H.-J. Schellnhuber, 2002: Global climate models violate scaling of the observed atmospheric variability. Phys. Rev. Lett., 89, 028501.

He, W., S. Zhao, Q. Liu, Y. Jiang, and B. Deng, 2016: Long-range correlation in the drought and flood index from 1470 to 2000 in eastern China. Int. J. Climatol., 36, 1676-1685.

Hurst, H. E., 1951: Long-term storage capacity of reservoirs. Trans. Amer. Soc. Civil Eng., 116, 770-799.

Hu, Q., D. Jiang, and G. Fan, 2014: Evaluation of CMIP5 models over Qinghai-Tibetan Plateau. Chinese J. Atmos. Sci., 38, 924-938 (in Chinese).

Immerzeel, W. W., L. P. Van Beek, and M. F. Bierkens, 2010: Climate change will affect the Asian water towers. Science,
328, 1382-1385.

Jacob, D. J., and D. A. Winner, 2009: Effect of climate change on air quality. Atmos. Environ., 43, 51-63.

Jiang, L., N. Li, Z. Fu, and J. Zhang, 2015: Long-range correlation behaviors for the $0-\mathrm{cm}$ average ground surface temperature and average air temperature over China. Theor. Appl. Climatol., 119, 25-31.

Jiang, L., N. Li, and X. Zhao, 2017: Scaling behaviors of precipitation over China. Theor. Appl. Climatol., 128, 63-70.

Huang, J., F. Zhang, L. Zhou, Z. Hu, and Y. Li, 2018: Regional changes of climate extremes and its effect on rice yield in Jiangsu province, southeast China. Environ. Earth Sci., 77, 106.

Kantelhardt, J. W., E. Koscielny Bunde, D. Rybski, P. Braun, A. Bunde, and S. Havlin, 2006: Long-term persistence and multifractality of precipitation and river runoff records. $J$. Geophys. Res. Atmos., 111, D01106.

Kantelhardt, J. W., E. Koscielny Bunde, H. H. Rego, S. Havlin, and A. Bunde, 2001: Detecting long-range correlations with detrended fluctuation analysis. Physica A: Stat. Mech. Appl., 295, 441-454.

Lennartz, S., and A. Bunde, 2011: Distribution of natural trends in long-term correlated records: A scaling approach. Phys. Rev., 84, 021129.

Masson, D., and R. Knutti, 2011: Spatial-scale dependence of climate model performance in the CMIP3 ensemble. $J$. Climate, 24, 2680-2692.

Monetti, R. A., S. Havlin, A. Bunde, and I. Applications, 2003: Long-term persistence in the sea surface temperature fluctuations. Physica A: Stat. Mech., 320, 581-589.

Parry, M. L., C. Rosenzweig, A. Iglesias, M. Livermore, and G. Fischer, 2004: Effects of climate change on global food production under SRES emissions and socio-economic scenarios. Global Environ. Change, 14, 53-67.

Peng, C. K., S. Havlin, M. Schwartz, and H. E. Stanley, 1991: Directed-polymer and ballistic-deposition growth with correlated noise. Phys. Rev., 44, R2239.

Peng, C. K., S. V. Buldyrev, S. Havlin, M. Simons, H. E. Stanley, and A. L. Goldberger, 1994: Mosaic organization of DNA nucleotides. Phys. Rev., 49, 1685.

Perkins, S., A. Pitman, N. Holbrook, and J. Mcaneney, 2007: Evaluation of the AR4 climate models' simulated daily maximum temperature, minimum temperature, and precipitation over Australia using probability density functions. $J$. Climate, 20, 4356-4376.

Song, F., and T. Zhou, 2014: Interannual variability of East Asian summer monsoon simulated by CMIP3 and CMIP5 AGCMs: Skill dependence on Indian Ocean-western Pacific anticyclone teleconnection. J. Climate, 27, 1679-1697.

Su, F., X. Duan, D. Chen, Z. Hao, and L. Cuo, 2013: Evaluation of the global climate models in the CMIP5 over the Tibetan Plateau. J. Climate, 26, 3187-3208.

Taylor, K. E., R. J. Stouffer, and G. A. Meehl, 2012: An overview of CMIP5 and the experiment design. Bull. Amer. Meteor. Soc., 93, 485-498.

Xu, X., C. Lu, X. Shi, and S. Gao, 2008: World water tower: An atmospheric perspective. Geophys. Res. Lett., 35.

Yuan, N., M. Ding, Y. Huang, Z. Fu, E. Xoplaki, and J. Luterbacher, 2015: On the long-term climate memory in the surface air temperature records over Antarctica: A nonnegligible factor for trend evaluation. J. Climate, 28, 5922-5934.

Zhao, S., and W. He, 2015: Evaluation of the performance of the Beijing Climate Centre Climate System Model 1.1 (m) to simulate precipitation across China based on long-range correlation characteristics. J. Geophys. Res. Atmos., 120, $12576-12588$.

Zhu, X., K. Fraedrich, Z. Liu, and R. Blender, 2010: A demonstration of long-term memory and climate predictability. $J$. Climate, 23, 5021-5029.

Manuscript received 11 December 2018, accepted 12 February 2019 SOLA: https://www.jstage.jst.go.jp/browse/solal 